(2) Open Access Full Text Article

REVIEW

\title{
Emerging role of dual antiplatelet therapy in the prevention of hepatitis B virus-associated hepatocellular carcinoma
}

This article was published in the following Dove Press journal:

Journal of Hepatocellular Carcinoma

13 November 2014

Number of times this article has been viewed

\author{
Roberto Aiolfi \\ Giovanni Sitia \\ Division of Immunology, \\ Transplantation and Infectious \\ Diseases, San Raffaele Scientific \\ Institute, Milan, Italy
}

Correspondence: Giovanni Sitia

San Raffaele Scientific Institute,

Division of Immunology,

Transplantation and Infectious Diseases,

Via Olgettina 58, 20I 32 Milan, Italy

Tel +3902 26434956

Fax +3902 26436822

Email sitia.giovanni@hsr.it
Abstract: Platelets, the chief effectors of vascular homeostasis, have been identified as important players in the pathogenesis of both acute and chronic liver disease in preclinical models of hepatitis B viral infection. Platelets are thought to promote the accumulation of virus-specific T-cells into the liver parenchyma. Importantly, the inhibition of platelet activation by clinically relevant doses of aspirin and clopidogrel was able to reduce immune-mediated necroinflammatory liver disease, extracellular matrix deposition, and hepatocellular carcinoma development; the same treatment was able to improve overall survival. These results strongly support the design of clinical trials aiming to define the potential of antiplatelet therapy in the prevention of hepatitis B virus-associated hepatocellular carcinoma.

Keywords: platelets, hepatocellular carcinoma, hepatitis B virus

\section{Introduction}

Hepatocellular carcinoma (HCC) is the fifth most common cause of death from cancer worldwide. ${ }^{1}$ Liver infection with the hepatitis B virus (HBV) is a major etiological factor for $\mathrm{HCC}$; this type of liver infection is responsible for over $50 \%$ of the reported cases of HCC. ${ }^{1}$ Among the described mechanisms by which HBV causes HCC, two are thought to be prominent: viral factors, such as the expression of viral gene products with procarcinogenic potential and insertional mutagenesis; and host factors linked to the antiviral immune response. ${ }^{2}$ In particular, HBV-associated chronic hepatitis, characterized by a dysfunctional virus-specific $\mathrm{CD} 8^{+} \mathrm{T}$-cell response that is unable to clear the virus from the infected hepatocytes, appears to play a major role in promoting continuous cycles of hepatocellular death and compensatory liver cell proliferation in the context of inflammatory mutagens that, over time, are thought to promote the multiple genetic alterations associated with $\mathrm{HCC}^{3}$

\section{Overview of current treatment options and patient outcomes}

Currently, several therapeutic approaches, mainly dictated by the stage of tumor progression, are available for the treatment of HCC. ${ }^{46}$ Apart from very early-stage HCC, for which surgical resection or liver transplantation represent a curative option, treatment and prognosis is poor for intermediate- and advanced-stage HCC patients, who represent more than $60 \%$ of those diagnosed with HCC. ${ }^{6,7}$ For cases in which surgical resection and liver transplantation are not possible, local ablation using radiofrequency or percutaneous ethanol injection and transarterial chemoembolization have become 
the most frequently used treatments, although they are often associated with high recurrence rates. ${ }^{6-9}$ Furthermore, other ablative therapies such as microwave therapy, cryoablation, ${ }^{6-9}$ and proton beam radiotherapy ${ }^{10}$ have proved to be somewhat effective in small-scale clinical trials and are valuable alternatives that need additional clinical evaluation. Finally, individuals with advanced-stage HCC may benefit from the multikinase inhibitor, sorafenib, although this has an improvement of median survival of only 7-8 weeks in spite of its high cost. ${ }^{11-13}$

Given the poor prognosis and the paucity of curative treatments for HCC, new strategies aimed to prevent the long-term consequences of chronic hepatitis are highly encouraged. With recent findings from a transgenic mouse model of chronic HBV, researchers were able to demonstrate the long-term use of dual antiplatelet therapy as a preventive strategy that is able to reduce the occurrence of $\mathrm{HCC}$ development. ${ }^{14}$

\section{The role of platelets and platelet- derived molecules in HCC development}

By using different mouse models of acute viral hepatitis, our group showed that virus-specific CTLs are key triggers for hepatic necroinflammatory liver disease during the course of HBV infection. ${ }^{3}$ In this process, platelets play an instrumental role. We illustrated this by taking advantage of two mouse models of self-limited viral hepatitis: HBV-replicating mice injected with HBV-specific CTLs; and mice infected with a hepatotropic adenovirus. Through these models, we showed that platelets can be detected at the site of liver damage, and that their depletion ameliorates liver disease by reducing the frequency and amount of virus-specific and virus-nonspecific inflammatory infiltrate recruited into the liver parenchyma. ${ }^{15,16}$

Importantly, we found that inhibitors of platelet activation (such as aspirin that blocks thromboxane $\mathrm{A}_{2}$ production by irreversibly acetylating cyclooxygenase 1 enzyme, and clopidogrel that inhibits the ADP P2Y purinoreceptor 12), were able to modulate the platelet-dependent intrahepatic accumulation of virus-specific CTL.

Since the oral administration of aspirin and clopidogrel is used in clinical settings for the treatment of patients with a high risk of thrombosis, we decided to evaluate the effects of the sustained inhibition of these two platelet activation pathways on the severity of chronic liver damage and HCC development in an established mouse model of immunemediated chronic hepatitis B. ${ }^{17}$ After the induction of acute hepatitis and until the end of the study, mice were treated with doses of aspirin and clopidogrel that are proportionate to the doses used in patients. These mice showed a significant reduction in the accumulation of virus-specific CTLs and of virus-nonspecific inflammatory cells within the liver; these mice also showed a consequential reduction of liver disease. ${ }^{14}$ Additionally, these mice displayed reductions in hepatocyte compensatory proliferation, collagen deposition (fibrosis), liver cell transformation, and HCC development. Importantly, treated animals showed a greater improvement in overall survival compared to the untreated mice. ${ }^{14}$ These results indicate that platelets not only influence the intrahepatic accumulation of HBV-specific CTLs during acute hepatitis, but are able to influence conditions during the chronic stages of the disease by modulating certain inflammatory reactions. These reactions are generated by the antiviral immune response against infected hepatocytes that promote HCC transformation during chronic HBV infection. ${ }^{3,18-20}$

Different possible mechanisms by which platelets could influence the recruitment of virus-specific CTLs have been proposed. Upon activation, platelets can express on their plasma membranes CD40 ligand, a molecule that is able to modulate different aspects of innate and adaptive immunity to contribute to the expansion and activity of CTLs that result in the accumulation of these cells at sites of viral replication. ${ }^{21-23}$ Furthermore, upon activation, platelet granule content is rapidly exocytosed to boost hemostasis, inflammation, and immune responses. Among these molecules, P-selectin and P-selectin glycoprotein ligand-1 have been known to play roles in the interactions between platelets, different subsets of leukocytes and the endothelial wall. Other molecules, such as platelet factor 4 , are able to activate and recruit cells at sites of inflammation. ${ }^{24}$ Furthermore, during chronic viral hepatitis and HCC development, other platelet-derived vasoactive molecules, such as serotonin, may play a role in the modulation of sinusoidal circulation, altering virus-specific CTL recirculation into the liver, ultimately contributing to liver damage. ${ }^{25,26}$

Mechanistically, at the dose used in our study, aspirin treatment alone inhibits the release of serotonin and other small molecules from $\delta$-granules with no effect on the release of a wide array of immunologically relevant proteins and peptides stored in the $\alpha$-granules that are instrumental in the functional cross-talk between platelets and leukocytes. ${ }^{22,24}$ Conversely, clopidogrel has been shown to downregulate the expression of these inflammatory molecules (P-selectin and CD40L); this process is able to inhibit a number of platelet-leukocyte interactions that link 
vascular injury to inflammatory reactions. ${ }^{27}$ In this scenario, the inhibition of different platelet activation pathways that is achieved by aspirin- and clopidogrel-combined therapy may ameliorate the course of immune-mediated chronic hepatitis and HCC progression through the distinct pharmacological effects of the two different drugs. The synergistic effect of aspirin and clopidogrel may reduce platelet influence in a complex pathogenic mechanism in which multiple pathways contribute to the intrahepatic accumulation of virus-specific CTLs. ${ }^{14,19,20}$

\section{Critical analysis of the potential role of antiplatelet therapy in the clinical setting}

Our preclinical study shows that inhibiting platelet activation by using two widely used antiplatelet drugs is able to ameliorate the development of HBV-induced immune-mediated $\mathrm{HCC}$, delay it, or both. ${ }^{14}$ Although the data presented in our study represent a step forward in the understanding of HBV-associated hepatocarcinogenesis, caution has to be taken before antiplatelet treatment may be applied in clinic. Since the HBV transgenic mouse model that is used does not support infection and viral replication, the outcome of combined antiplatelet therapy on these parameters should be carefully monitored. ${ }^{14}$ The antiplatelet therapy-mediated reduction of virus-specific CTL accumulation into the liver may have negative effects on the control of HBV viremia. Simultaneous use of nucleoside and nucleotide inhibitors might be supportive in limiting virus replication in patients subjected to antiplatelet therapy. Moreover, since the transgenic mouse model used in the preclinical study lacks the expression of the whole HBV genome, there is the potential to underestimate the role of viral gene products with transforming potential in HCC development during HBV chronic infections. For these reasons, additional preclinical work using the woodchuck hepatitis virus model may give further information on viral replication, liver disease severity, and oncogenic potential in the context of combined antiplatelet therapy. Finally, the increased risk of bleeding might limit the long-term use of aspirin and clopidogrel in some patients affected by chronic liver diseases. However, the findings from a recent report suggest that patients with thrombosis may be at higher risk and, therefore, might benefit from this approach. ${ }^{28}$ In addition, the long-term use of aspirin was recently shown to reduce the risk of $\mathrm{HCC}$ in patients suffering from chronic liver disease of unknown etiologies. ${ }^{29}$ These observational results, together with our preclinical work, strongly support the basis for future clinical trials aimed at evaluating the effect of single or combined antiplatelet therapy in the prevention of HCC development in HBV-infected patients.

\section{Highlights}

- Platelets promoted the accumulation of virus-specific CTLs into the liver parenchyma in mouse models of acute and chronic hepatitis B infection.

- Long-term treatment with aspirin and clopidogrel reduced platelet-dependent accumulation of virus-specific CTLs and consequently reduced liver inflammation.

- In mouse models of immune-mediated chronic hepatitis B, continuous administration (approximately 600 days of treatment) of aspirin and clopidogrel diminished the severity of persistent liver damage and of liver fibrosis without causing significant side effects. These effects prevented HCC development and improved overall survival.

- Drugs targeting platelet functionality may present a novel preventive strategy for patients with chronic HBV infection who are at risk of developing liver cancer.

\section{Acknowledgment}

This work was partially supported by grant GR08.17 from the Italian Ministry of Health.

\section{Disclosure}

The authors report no conflicts of interest in this work.

\section{References}

1. Nguyen VT, Amin J, Law MG, Dore GJ. Predictors and survival in hepatitis B-related hepatocellular carcinoma in New South Wales, Australia. J Gastroenterol Hepatol. 2009;24(3):436-442.

2. Ganem D, Prince AM. Hepatitis B virus infection - natural history and clinical consequences. N Engl J Med. 2004;350(11):1118-1129.

3. Guidotti LG, Chisari FV. Immunobiology and pathogenesis of viral hepatitis. Annu Rev Pathol. 2006;1:23-61.

4. Bruix J, Llovet JM. Prognostic prediction and treatment strategy in hepatocellular carcinoma. Hepatology. 2002;35(3):519-524.

5. Llovet JM, Fuster J, Bruix J. Prognosis of hepatocellular carcinoma. Hepatogastroenterology. 2002;49(43):7-11.

6. European Association For The Study Of The Liver; European Organisation For Research And Treatment Of Cancer. EASL-EORTC clinical practice guidelines: management of hepatocellular carcinoma. J Hepatol. 2012;56(4):908-943.

7. Bruix J, Sherman M; American Association for the Study of Liver Diseases. Management of hepatocellular carcinoma: an update. Hepatology. 2011;53(3):1020-1022.

8. Cho YK, Kim JK, Kim MY, Rhim H, Han JK. Systematic review of randomized trials for hepatocellular carcinoma treated with percutaneous ablation therapies. Hepatology. 2009;49(2):453-459.

9. Livraghi T, Meloni F, Di Stasi M, et al. Sustained complete response and complications rates after radiofrequency ablation of very early hepatocellular carcinoma in cirrhosis: Is resection still the treatment of choice? Hepatology. 2008;47(1):82-89.

10. Bush DA, Kayali Z, Grove R, Slater JD. The safety and efficacy of high-dose proton beam radiotherapy for hepatocellular carcinoma: a phase 2 prospective trial. Cancer. 2011;117(13):3053-3059. 
11. Llovet JM, Ricci S, Mazzaferro V, et al; SHARP Investigators Study Group. Sorafenib in advanced hepatocellular carcinoma. NEngl J Med. 2008;359(4):378-390.

12. Cheng AL, Kang YK, Chen Z, et al. Efficacy and safety of sorafenib in patients in the Asia-Pacific region with advanced hepatocellular carcinoma: a phase III randomised, double-blind, placebo-controlled trial. Lancet Oncol. 2009;10(1):25-34.

13. Hsu C, Shen YC, Cheng AL. Sorafenib for the treatment of hepatocellular carcinoma across geographic regions. Expert Rev Clin Pharmacol. 2009;2(2):129-136.

14. Sitia G, Aiolfi R, Di Lucia P, et al. Antiplatelet therapy prevents hepatocellular carcinoma and improves survival in a mouse model of chronic hepatitis B. Proc Natl Acad Sci U S A. 2012;109(32):E2165-E2172.

15. Iannacone $M$, Sitia $G$, Isogawa $M$, et al. Platelets mediate cytotoxic T lymphocyte-induced liver damage. Nat Med. 2005;11(11): 1167-1169.

16. Iannacone M, Sitia G, Narvaiza I, Ruggeri ZM, Guidotti LG. Antiplatelet drug therapy moderates immune-mediated liver disease and inhibits viral clearance in mice infected with a replication-deficient adenovirus. Clin Vaccine Immunol. 2007;14(11):1532-1535.

17. Nakamoto Y, Kaneko S. Analysis of intrahepatic lymphocyte subsets in a transgenic mouse model of immune-mediated hepatocarcinogenesis. J Exp Clin Cancer Res. 2006;25(1):55-57.

18. Bertoletti A, Maini MK. Protection or damage: a dual role for the virus-specific cytotoxic $\mathrm{T}$ lymphocyte response in hepatitis $\mathrm{B}$ and $\mathrm{C}$ infection? Curr Opin Microbiol. 2000;3(4):387-392.

19. Sitia G, Iannacone M, Guidotti LG. Anti-platelet therapy in the prevention of hepatitis B virus-associated hepatocellular carcinoma. J Hepatol. 2013;59(5):1135-1138.
20. Sitia G. Platelets promote liver immunopathology contributing to hepatitis B virus-mediated hepatocarcinogenesis. Semin Oncol. 2014; 41(3):402-405.

21. Elzey BD, Tian J, Jensen RJ, et al. Platelet-mediated modulation of adaptive immunity. A communication link between innate and adaptive immune compartments. Immunity. 2003;19(1):9-19.

22. Elzey BD, Sprague DL, Ratliff TL. The emerging role of platelets in adaptive immunity. Cell Immunol. 2005;238(1):1-9.

23. Iannacone M, Sitia G, Isogawa M, et al. Platelets prevent IFN-alpha/ beta-induced lethal hemorrhage promoting CTL-dependent clearance of lymphocytic choriomeningitis virus. Proc Natl Acad Sci U S A. 2008;105(2):629-634.

24. Semple JW, Italiano JE Jr, Freedman J. Platelets and the immune continuum. Nat Rev. Immunol. 2011;11(4):264-274.

25. Lang PA, Contaldo C, Georgiev P, et al. Aggravation of viral hepatitis by platelet-derived serotonin. Nat Med. 2008;14(7):756-761.

26. Soll C, Jang JH, Riener MO, et al. Serotonin promotes tumor growth in human hepatocellular cancer. Hepatology. 2010;51(4): 1244-1254.

27. Smyth SS, Reis ED, Zhang W, Fallon JT, Gordon RE, Coller BS. Beta(3)-integrin-deficient mice but not P-selectin-deficient mice develop intimal hyperplasia after vascular injury: correlation with leukocyte recruitment to adherent platelets 1 hour after injury. Circulation. 2001;103(20):2501-2507.

28. Tripodi A, Mannucci PM. The coagulopathy of chronic liver disease. N Engl J Med. 2011;365(2):147-156.

29. Sahasrabuddhe VV, Gunja MZ, Graubard BI, et al. Nonsteroidal anti-inflammatory drug use, chronic liver disease, and hepatocellular carcinoma. J Natl Cancer Inst. 2012;104(23):1808-1814.
Journal of Hepatocellular Carcinoma

\section{Publish your work in this journal}

The Journal of Hepatocellular Carcinoma is an international, peerreviewed, open access journal that offers a platform for the dissemination and study of clinical, translational and basic research findings in this rapidly developing field. Development in areas including, but not limited to, epidemiology, vaccination, hepatitis therapy, pathology and

\section{Dovepress}

molecular tumor classification and prognostication are all considered for publication. The manuscript management system is completely online and includes a very quick and fair peer-review system, which is all easy to use. Visit http://www.dovepress.com/testimonialsphp to read real quotes from published authors. 